\title{
Engaging the public in healthcare decision-making: results from a Citizens' Jury on emergency care services
}

\author{
P A Scuffham, ${ }^{1}$ N Moretto, ${ }^{1}$ R Krinks, ${ }^{2}$ P Burton, ${ }^{3}$ J A Whitty, ${ }^{1,4}$ A Wilson, ${ }^{5}$ \\ $G$ Fitzgerald, ${ }^{6} \mathrm{P}$ Littlejohns, ${ }^{7}$ E Kendall ${ }^{2}$
}

${ }^{1}$ Centre for Applied Health Economics, Menzies Health Institute Queensland, Griffith University, Meadowbrook, Queensland, Australia

${ }^{2}$ Centre of National Research on Disability and

Rehabilitation, Menzies Health Institute Queensland, Griffith University, Meadowbrook, Queensland, Australia ${ }^{3}$ Urban Research Program, Griffith School of Environment, Griffith University, Southport, Queensland, Australia ${ }^{4}$ School of Pharmacy, Faculty of Health and Behavioural Sciences, University of Queensland, St Lucia, Queensland, Australia ${ }^{5}$ Menzies Centre for Health Policy, School of Public Health, University of Sydney, Sydney, New South Wales, Australia ${ }^{6}$ School of Public Health, Institute of Health and Biomedical Innovation, Queensland University of Technology, Brisbane, Queensland, Australia ${ }^{7}$ Division of Health and Social Care Research, Faculty of Life Sciences and Medicine, King's College London, London, UK

\section{Correspondence to}

Professor P A Scuffham, Centre for Applied Health Economics, School of Medicine, Griffith University, Logan Campus, Meadowbrook, QLD 4131, Australia;

p.scuffham@griffith.edu.au

Received 24 December 2015 Revised 9 May 2016 Accepted 30 May 2016 Published Online First 20 June 2016

\section{CrossMark}

To cite: Scuffham $P A$, Moretto N, Krinks $\mathrm{R}$, et al. Emerg Med J 2016;33:782788.

\section{ABSTRACT}

Background Policies addressing ED crowding have failed to incorporate the public's perspectives; engaging the public in such policies is needed.

Objective This study aimed at determining the public's recommendations related to alternative models of care intended to reduce crowding, optimising access to and provision of emergency care.

Methods A Citizens' Jury was convened in Queensland, Australia, to consider priority setting and resource allocation to address ED crowding. Twenty-two jurors were recruited from the electoral roll, who were interested and available to attend the jury from 15 to 17 June 2012. Juror feedback was collected via a survey immediately following the end of the jury.

Results The jury considered that all patients attending the ED should be assessed with a minority of cases diverted for assistance elsewhere. Jurors strongly supported enabling ambulance staff to treat patients in their homes without transporting them to the ED, and allowing non-medical staff to treat some patients without seeing a doctor. Jurors supported (in principle) patient choice over aspects of their treatment (when, where and type of health professional) with some support for patients paying towards treatment but unanimous opposition for patients paying to be prioritised. Most of the jurors were satisfied with their experience of the Citizens' Jury process, but some jurors perceived the time allocated for deliberations as insufficient.

Conclusions These findings suggest that the general public may be open to flexible models of emergency care. The jury provided clear recommendations for direct public input to guide health policy to tackle ED crowding.

\section{INTRODUCTION}

ED crowding is a serious and growing international public health issue, ${ }^{1}$ fuelled by a global trend of increasing demands on the health systems and a lack of access to inpatient beds (access block). ${ }^{2} \mathrm{ED}$ crowding can lead to poor patient outcomes. Several countries have introduced strategies such as time-based targets aimed at ameliorating ED crowding; however, the strategies have not been fully successful in solving this complex problem. ${ }^{3}$

To date, policy measures to address ED crowding have often failed to incorporate the public's perspectives as public input is often not sought or, if it is sought, it is disregarded. ${ }^{4}$ Public engagement to inform healthcare decision-making has been recognised as a key ingredient in modern policy-making

\section{Key messages}

What is already known on this subject?

- Efficient and responsive policy that considers the public's perspective is needed to address the complex and serious issue of ED crowding.

- There is limited research on public preferences for priority setting and resource allocation to optimise access to and provision of emergency care.

What might this study add?

- Our Citizens' Jury provided clear recommendations for direct public input for policy decision-making.

- Our findings suggest that the general public may be open to flexible models of emergency care.

and may even increase the likelihood of the policy being more effectively implemented. ${ }^{5}$ A Citizens' Jury is a well-accepted, deliberative method of public engagement which elicits public views around specific topics, including health policy. ${ }^{67}$ This approach is particularly useful for informing complex policy decisions, such as those involving priority setting and resource allocation. ${ }^{5}$ While the UK's National Institute for Health and Care Excellence (NICE) routinely employs a modified Citizens' Jury process for assistance in priority setting, support for Citizens' Juries is growing in Australia and other countries. ${ }^{8} 9$ The use of Citizens' Juries to foster community engagement, especially in the area of allocation of limited resources in the context of competing priorities, have been recommended by governments. ${ }^{10}$ This is mirrored in policy-requiring hospitals and health services to consult with members of the community about the provision of health services. ${ }^{11}$

In a Citizens' Jury, a group of randomly selected individuals that demographically reflect the wider community is convened to examine a prespecified topic. Expert witnesses present evidence to the jury, which forms the basis of the jury deliberations. The verdicts and recommendations are thought to broadly represent the values and intellect of the wider community. ${ }^{8} 91213$ This approach can directly impact policy, making Citizen Juries an invaluable community engagement approach for efficient and responsive policy. ${ }^{7}{ }^{14}$ 
There is limited research on public preferences for strategies to optimise the use of EDs and the allocation of resources to address crowding. Engaging the public in the design, promotion and implementation of strategies to address ED crowding is, therefore, needed. To address this issue, we conducted a Citizens' Jury to determine the public's preferences related to potential changes to the model of care intended to reduce crowding, optimising access to and provision of emergency care. This paper describes the findings and recommendations of the Citizens' Jury focusing on priority setting and resource allocation to tackle the problem of ED crowding within the Australian healthcare system. This study on the ED is part of a larger study described elsewhere. ${ }^{15}$

\section{METHOD}

\section{Recruitment and selection}

We used a combination of random and purposive sampling to recruit participants to this study. A random sample of 2000 people were selected from the electoral roll covering the Queensland Health Metro-South Health Service District (a large metropolitan health service in southeast Queensland that caters for 250000 ED cases across five hospitals annually), were sent a letter of invitation, an information sheet and a screening survey. The screening survey included interest and availability in participating, sociodemographic questions (including age, gender, income, employment status, country of birth, language, indigenous heritage, private health insurance status and health concession card possession), affiliations with special interest groups and occupation. Participants were eligible to participate if they were aged at least 18 years and were willing and available to participate in the Citizens' Jury. Exclusion criteria were affiliation with a special interest group such as a patient advocacy group, employed as a healthcare professional, or ever worked in an ED or an emergency care service. A sitting fee for the jurors (\$A300) and travel and accommodation expenses were offered to reduce volunteer bias.

Of the 490 screening surveys returned, 204 (42\%) were interested and available to participate. Of these, $37(18 \%)$ were excluded based on the prespecified exclusion criteria. From the remaining sample of 167 respondents, 22 eligible respondents were purposively selected to be on the jury stratified to match the demographic profile of Queensland. Of the 22 respondents invited to be jurors, 18 agreed to participate. Seven replacement jurors were invited, and six accepted, leaving a jury of 22 with 2 alternate jurors. The sample size of 22 jurors is considered large enough to obtain informed public opinion as it lies between the number of citizens that typically sit on a legal jury (12 people) and on the UK's NICE Citizen Council (30 people). The jurors were mailed an information sheet, a discrete choice preference survey (findings to be reported as part of the overall discrete choice experiment) and a consent form, which was signed and returned prior to the start of the Citizens' Jury. The jurors were then mailed information about the Citizens' Jury and relevant background information on EDs.

\section{Questions for deliberation by the jury}

The questions put to the Citizens' Jury were developed based on information from a literature review, scoping meetings with stakeholders, a focus group with ED clinicians and other stakeholders, and input from research partners. One overarching, six subquestions, and one additional question about emergency care services were put to the jury for consideration (box 1).

\section{Citizens' Jury process}

The Citizens' Jury was convened on the 15-17 June 2012 in Brisbane, Queensland, Australia. It was conducted in line with the standardised Citizens' Jury procedure proposed by the Jefferson Center, ${ }^{12}$ including juror and witness selection, question development, planned hearings, deliberative periods for discussion and compilation of recommendations. At the outset, the jurors were informed that Queensland Health and the Southern Adelaide Local Health Network had sponsored the study and that the purpose of the jury was to make recommendations to both organisations on what they considered to be acceptable approaches to managing the ED given the crowding issues. This would potentially have a direct impact on healthcare policy. They were told that their recommendations would be compiled into a report and presented to senior management in both organisations.

During the three consecutive days, jurors listened to 12 expert witnesses reflecting a wide range of stakeholders across six evidence and two panel sessions on the topic of optimising access to and provision of emergency care (box 2). Jurors questioned the witnesses and clarified the evidence presented to them. Any unanswered questions were followed-up with witnesses and responses were relayed to jurors.

Jurors engaged in four deliberative discussion periods with the two independent facilitators with the witnesses absent. During these deliberations, jurors engaged in activities in plenary and small groups (eg, round robin, voting) aimed at helping them to ensure good deliberative discussions, prevent conflict among jurors, reflect on witness testimony and prioritise their preferences (box 2). If any disagreements among jurors occurred, the facilitator explored the issue in more detail and amended the juror responses to reflect the common underlying viewpoint of the jurors. On Day 3, the jurors drafted, reviewed and revised the verdicts and recommendations. The final typed

Box 1 Questions about emergency care services put to the jury

Charge question

The ED should treat everyone who presents.

Subquestions

- Are there circumstances where it is acceptable to not treat someone presenting at the ED?

- Should patients be given a choice over when they are treated, where they are treated and by whom (type of health professional)?

- Is it acceptable for patients to be treated by non-medical staff such as paramedics, nurses and allied health professionals without seeing a doctor?

- Should paramedics be enabled to treat patients in their home without bringing them to the ED?

- Are there any circumstances that patients should pay towards the cost of treatment?

- Should patients with minor illnesses or injuries have a choice to pay to be seen in a priority queue?

Additional question

- What would make the biggest difference to improving the efficiency, effectiveness and optimal use of EDs? 


\section{Box 2 Summary of witness sessions and deliberations}

\section{Day 1}

Introduction

Jury welcome and induction—facilitator/s (135 min)

Session 1

Topic: EDs in Australia-researcher/policy expert (45 min)

An overview of EDs within the Australian health system.

Session 2

Topic: How an ED functions-nurse unit manager (60 min)

Day 2

An overview of how $E D$ s function.

Session 3

Topic: Issues and priorities in EDs—director of an ED (45 min)

Information about how the ED interacts with the rest of the hospital.

Session 4

Panel—nurse (bed management); paediatrician (retrieval); ambulance driver (60 min)

Panellists responded to the charge and subquestions from their own perspective.

Deliberation (70 min)

Round robin exercise: jurors to express their views on the questions.

Voting preferences: juror voted on each question using visual analogue scales (VAS) hung on the wall anchored with labels at each end (strongly oppose and strongly support).

Jurors were asked to indicate which two questions most interested them.

Session 5

Consumer panel (advocates for mental health, mothers/babies, carers) $(60 \mathrm{~min})$

Consumer panellists provided information from their own perspective.

Deliberation (four groups) (30 $\mathrm{min})$

Jurors deliberated on what would make the most positive impact on efficiency, effectiveness and appropriate use of EDs?

Session 6

Topic: emergency nurse practitioners-nurse practitioner (30 min)

An overview of role and training of emergency nurse practitioners and efficacy data.

Session 7

Topic: Primary care-general practitioner (GP) (30 min)

An overview of the role of primary health care, a model of primary care and ED care.

Deliberation (four groups) (90 min)

Day 3

Each group responded to one of the top four questions of most interest (Questions 2-5).

Session 8

Topic: Summary—researcher/policy expert (45 min)

An overview of emergency care services in Australia and a summary of the testimony.

Deliberation (plenary and small groups)

Jurors listed top three strategies that would make the most positive impact on use of EDs.

For each question, jurors adjusted voting preferences, wrote comments and indicated their endorsement (5 'ticks' per question) with jury's comments.

Jurors drafted $(75 \mathrm{~min})$ and finalised $(75 \mathrm{~min})$ verdicts and recommendations.

version was displayed on a data projector and final edits were made. The jurors confirmed the final version accurately reflected their views. Jurors attended a debriefing feedback session and completed an evaluation survey.

\section{Data collection and analysis}

With minimal direction from the facilitators, jurors drafted the final verdicts and recommendations. Juror feedback about the Citizens' Jury process was collected from a jury debriefing session, and a 17-item evaluation survey covering factors such as time allocated for deliberations, the duration of the jury, information presented and overall satisfaction with the process. A $\mathrm{PhD}$ student (RK) collected additional information on consumer voices using diaries provided to the jurors; this will be reported separately. Jurors' feedback during the debriefing was audio- recorded and transcribed. Feedback comments from the jurors' diaries were compiled. Satisfaction ratings in the survey used a five-point Likert scale (ranging from very dissatisfied to very satisfied) regarding the juror's satisfaction with the Citizens' Jury process. A thematic analysis of the qualitative data from all three sources was performed to identify emergent themes.

\section{RESULTS}

\section{Description of jury participants}

Members of the jury came from a broad range of age groups, place of residence and occupations. Table 1 shows that the age and sex distribution of the jury broadly reflects the Queensland population; women (aged 35 to 54 years) and men (aged 55 years and over) were slightly over-represented and 
Table 1 Age and sex of the Citizens' Jury and the Queensland population

\begin{tabular}{|c|c|c|c|c|c|c|}
\hline \multirow[b]{2}{*}{ Age group (years) } & \multicolumn{3}{|c|}{ Citizens' Jury (\%) } & \multicolumn{3}{|c|}{$\begin{array}{l}\text { Queensland population } \\
(\%)^{16}\end{array}$} \\
\hline & Female & Male & Total & Female & Male & Total \\
\hline $18-34$ & 18 & 14 & 32 & 15 & 15 & 30 \\
\hline $35-54$ & 23 & 18 & 41 & 19 & 18 & 37 \\
\hline $55+$ & 18 & 9 & 27 & 17 & 16 & 33 \\
\hline Total & 59 & 41 & 100 & 51 & 49 & 100 \\
\hline
\end{tabular}

under-represented in the jury, respectively, when compared with the Queensland population.

The profile of the jury was comparable on numerous relevant demographic characteristics to the profile of the Queensland population (table 2).

\section{Jury's verdicts to the charge and subquestions}

The jury's verdicts to the charge and subquestions including additional recommendations are detailed below (Day 3, $\mathrm{n}=22$; box 3). Overarching question: The jury recommended that all patients attending EDs should be assessed, with a minority of cases diverted for assistance elsewhere (eg, to their General Practitioner (GP) or their social worker), if appropriate. The jurors considered that assessment of a patient was part of treatment (ie, determining that no medical intervention was required). Subquestion 1: The Jury considered there were very limited instances when it is acceptable to refer patients elsewhere such as when patients were too violent. Subquestion 2: The majority of jurors supported in principle, with some dissent, that patients with the capacity to make an informed choice over aspects of their treatment (when, where and type of health professional) should be given the option to do so. Jurors believed patient choice was important, yet highlighted the need for guidelines to inform the process. Subquestion 3: The jury strongly agreed that it is acceptable for patients with non-life-threatening conditions and minor injuries (Australasian Triage Scale categories 3-5) and uncomplicated pregnancies to be treated by appropriate non-medical staff without consulting a medical practitioner. Subquestion4: The jury strongly agreed that paramedic staff should be enabled to assess and treat consenting patients in their homes or at the site, without transporting them to the ED (provided the patient is stable, confident, capable of self-management and understands their options). For this to occur, jurors recommended introducing policies, legislation, administrative structures, training (including psychiatric and paediatric) and technology (eg, telemedicine linked to medical practitioners) to support paramedics in this extended care role. Subquestion 5: The jurors displayed diverging views, with tempered support, that patients should pay towards the costs of treatment under some circumstances (eg, if additional costs are incurred due to patients' requests). Subquestion 6: All jurors strongly opposed patients with minor illnesses or injuries having a choice to pay to be seen in a priority queue.

\section{Rationale for the jury's verdicts}

The main driver behind the jury passing these verdicts was their understanding of the critical issues facing EDs in Australia and

Table 2 Demographic characteristics of the Citizens' Jury and the Queensland population

\begin{tabular}{|c|c|c|c|}
\hline \multirow[b]{2}{*}{ Demographic characteristics } & \multicolumn{2}{|c|}{ Citizens' Jury } & \multirow{2}{*}{$\begin{array}{l}\text { Queensland } \\
\text { population } \\
\%\end{array}$} \\
\hline & $\mathbf{n}$ & $\%$ & \\
\hline Born overseas ${ }^{16}$ & 4 & 18 & 21 \\
\hline Speak a language other than English at home ${ }^{16}$ & 2 & 9 & 10 \\
\hline Indigenous $^{16}$ & 1 & 5 & 4 \\
\hline Have private health insurance ${ }^{17}$ & 11 & 50 & 48 \\
\hline $\begin{array}{l}\text { Have a concession card (Centrelink Business Integrity \& Workflow Systems } \\
\text { Branch. Concession card customers by federal electorate (Unpublished data). } \\
\text { Canberra: Strategic Performance \& Information Management Branch Centrelink, } \\
\text { 2012). }\end{array}$ & 4 & 18 & 25 \\
\hline \multicolumn{4}{|l|}{ Highest educational attainment ${ }^{18}$} \\
\hline Up to year 12 & 12 & 54 & 51 \\
\hline Diploma or trade certificate & 5 & 23 & 28 \\
\hline Bachelor's degree or higher & 5 & 23 & 21 \\
\hline \multicolumn{4}{|l|}{ Employment $^{16}$} \\
\hline Full time & 8 & 36 & 45 \\
\hline Part time & 4 & 18 & 18 \\
\hline Not in labour force & 8 & 36 & 33 \\
\hline Unemployed & 2 & 9 & 5 \\
\hline \multicolumn{4}{|l|}{ Occupation of those in labour force, $n=14^{19}$} \\
\hline Manager and professionals & 5 & 36 & 32 \\
\hline Technicians and trades & 2 & 14 & 15 \\
\hline Community and personal services; clerical and administrative; sales & 6 & 43 & 34 \\
\hline Machinery operators; drivers and labourers & 1 & 7 & 18 \\
\hline \multicolumn{4}{|l|}{ Annual household income ${ }^{16}$} \\
\hline$<\$ A 50000$ & 7 & 32 & 30 \\
\hline$\$ A 50000-\$ A 200000$ & 14 & 64 & 65 \\
\hline$>\$ A 200000$ & 1 & 5 & 5 \\
\hline
\end{tabular}




\section{Box 3 Verdicts to the questions put to the jury and recommendations}

Jury's verdicts and recommendations

Verdicts

Charge:

The ED should treat everyone who presents.

Verdict: support; all patients should be assessed

\section{Subquestions}

- Are there circumstances where it is acceptable to not treat someone presenting at the ED?

Verdict: very strong support

- Should patients be given a choice over when they are treated, where they are treated and by whom (type of health professional)? Verdict: supported in principle

- Is it acceptable for patients to be treated by non-medical staff such as paramedics, nurses and allied health professionals without seeing a doctor?

Verdict: very strong support

- Should ambulance staff be enabled to treat patients in their home without bringing them to the ED?

Verdict: very strong support

- Are there any circumstances that patients should pay towards the cost of treatment?

Verdict: diverging views, with tempered support

- Should patients with minor illnesses or injuries have a choice to pay to be seen in a priority queue?

Verdict: no (unanimous)

Recommendations to achieve the greatest impact on improving the efficiency, effectiveness and optimal use of EDs

Short-term strategies

- Expand the roles and responsibilities of nurses

- Allow paramedics to treat patients on-site

- Provide the legislative and organisational support for enhanced care roles

- Introduce transit lounges between EDs and hospital wards

- Improve transfer and handover procedures between paramedics and ED staff, ED and ward staff and between and within disciplines

- Increase the frequency of hospital rounds to improve discharge rates

- Provide better community care options

- Educate the public about self-care, first aid, health, function of EDs, alternative services, private EDs

- Improve access to patients' medical histories (to help assess patient capacity)

- Sign a disclaimer (by patients)

- Provide free transport home for patient transported to an ED >20 km

Long-term strategies

- Cover treatment costs for private patients attending private EDs

- Improve design of EDs for privacy (eg, private areas for triage)

- Authorise paramedics to determine whether to transport patients to the ED or super clinic

- Improve flexibility in Medicare funding options for private clinics and private EDs

- Introduce financial incentives for private ownership of clinics

- Introduce incentives for general practitioners (GPs) to provide minor procedures/wound care

- Explore alternative care options on-site or off-site (24 hour super clinics, after-hours GP clinics)

- Develop 'best practice' systems or processes through research with trial hospitals

the importance of improving the efficiency of the system. The jury believed that the majority of patients presenting to the ED are legitimately ill or injured and felt that any changes to the system should have an emphasis on patient autonomy, safety and confidence through the provision of quality and culturally sensitive emergency care. The jurors insisted on appropriate training, resources and support systems to treat patients effectively and mitigate against potential litigation. Jurors held a strong belief that public EDs and any clinical-based treatment decisions should remain free of charge in the Australian Public Health System.

\section{Juror feedback and emerging themes}

At the end of the jury, most of the jurors were satisfied or very satisfied with the Citizens' Jury in general and as an unbiased method to elicit their views, but a substantial minority were not satisfied with the amount of time allocated to the various components. Juror's comments indicated that they would have preferred more time for witness and deliberative sessions, more panel sessions, more frequent diary moments and the ability to recall witnesses.

A thematic analysis of the qualitative data on the jury process identified four themes: the value of the Citizens' Jury as a model, improved understanding of EDs, personal growth and consensus among a diverse group. Jurors' comments indicate that they found the jury experience to be positive and thought Citizens' Juries were a good model for public engagement. For many jurors, their involvement in the jury improved their understanding and changed their perceptions of EDs and the health system, and also formed part of a broader learning experience. Many jurors were surprised that agreement could be reached among such a diverse group. 


\section{DISCUSSION}

After deliberating the evidence on EDs, our Citizens' Jury found that the ED should assess, but not necessarily treat, all presenting ED patients. The jury supported a multifaceted and community-based approach to divert a minority of patients to alternative care services for treatment, if appropriate and available. To this end, the jury supported the provision of improved community care and funding options to better sustain patients in their homes and increase use of alternative cost-effective services. These recommendations are consistent with those of the Australasian College for Emergency Medicine (ACEM) ${ }^{20}$ and are in line with the literature showing that countries with effective healthcare services outside the ED report less ED crowding. ${ }^{1}$ Diverting a minority of patients would not be a suitable strategy in countries, such as the USA and The Netherlands, which have legal obligations to treat all patients. ${ }^{13}$ As most initiatives have a marginal effect, if any, on relieving ED patient capacity pressure, ${ }^{21}$ more research is needed to establish which models of care are clinically effective in addressing ED crowding. The jury believe public education is essential in order for members of the public to shift their existing views of EDs and embrace fundamental changes to the system. Such education should be directed to patients and also the wider community as the decision to attend an ED can equally fall on either party. ${ }^{22}$ The impact of education on ED demand is unknown; however, it is encouraging to note that educating the jurors, who themselves are ordinary citizens, resulted in them having a better understanding of the issues that often plague EDs and the health system and an appreciation for the need for innovative and flexible solutions.

The jury's strong support for enabling adequately trained ambulance staff to treat a subgroup of patients in their homes, at the scene or transfer them to alternative care serviceswithout transporting them to the ED-are largely consistent with previous studies that suggest that the public are supportive of such initiatives. ${ }^{22}$ With a significant number of all public hospital ED presentations arriving by an ambulance service, the majority of which are not emergencies or resuscitations, ${ }^{23}$ using paramedics in these enhanced roles has the potential to moderate the growth in demand for ED services. ${ }^{24}$ In Denmark, where ED crowding is not a major problem, ambulances are staffed by nurse anaesthetists or physicians who are authorised to treat and discharge patients at the scene. ${ }^{1}$

This alternative care model to the existing inflexible transportfocussed paramedic service may gain broad support from the community, and if found to be safe and effective without compromising response times and patient safety, it has the potential to reduce ED presentations and help ameliorate ED crowding.

The jury's strong support for enhancing the roles of non-medical staff to treat patients with non-life-threatening conditions/injuries-without a medical consultation-is largely consistent with previous studies that suggest that the public support expanded roles for non-medical personnel, if safe to do so. ${ }^{22}$ As the vast majority of all public hospital ED presentations considered non-emergency cases, ${ }^{23}$ increasing the scope of all non-medical health professionals may prove worthwhile; however, the impact on staff workload would need to be managed. The jury's recommendation to introduce whole-of-hospital strategies in the form of effective transfer and handover procedures, frequent hospital discharge rounds and transit lounges (an interim waiting area for patients such as waiting for bed allocation on admission) are in line with those identified in the international literature to address access block. $^{1} 320$ Furthermore, the transit lounges, which have already been introduced in some hospitals in Australia, may gain support from the broader public for their continued use or expansion into other hospitals.

The jury's general support for greater patient choice over treatment reflects the health sector's commitment to patient-centred care; yet, how this would work in practice remains unknown. The Australian healthcare system is a mixed public and private system; EDs are predominantly located in public hospitals although some private hospitals operate an ED for fee-paying patients. While there may be concerns that co-payment models for EDs may unknowingly decrease the ED presentations of those requiring emergency care, the jury's support for patients contributing to the cost of treatment under limited circumstances may be more appropriate (eg, where a patient requests ambulance staff to take them to an ED that is not the nearest appropriate ED). The jury's unanimous opposition to patients paying for prioritised treatment reflects the fundamental values of fair and equitable emergency care. The jury's support for crucial research to develop 'best practice' systems or processes is echoed by the ACEM's urgent request for an evidence base of interventions to inform funding decisions. ${ }^{2}$

This study demonstrates a novel approach to eliciting public preferences around the complex healthcare issue of ED crowding. These findings set the framework for public support for changes to policy around alternative models of emergency care and more flexible models of service delivery outside the ED, especially those with very strong support (eg, non-medical staff providing treatment) that are easy to implement and costeffective. This study reports on the first healthcare Citizens' Jury held in Queensland, Australia, and the methods aligned with the Jefferson Center's classic model of a Citizens' Jury. As such, this study may serve to impact the processes of public participation in healthcare prioritisation in Australia and internationally.

This study has several limitations. Selecting jurors from a sample that were willing and available to sit on the jury and that resided in the same geographical area may have produced an unintended selection bias not evident in the sample demographic characteristics such that the jury's views may not have been fully representative of the broader Queensland population. The verdicts reached by this group of citizens may differ from patients who are acutely ill or injured seeking emergency care; however, there is emerging research that patients are accepting of alternative models of care. ${ }^{22}$ It remains unknown whether this approach would generate similar verdicts in members of the public outside of a group context and in policy-makers. Time constraints may also have played a role in the facilitator putting disagreements between jurors aside rather than dealing with them at the time. It is possible that the jury may have been more influenced by some of the witness testimony than others and that the verdicts and recommendations from this small group of citizens may not reflect the views of the broader public. With the ED and elective surgery units competing for limited resources and funding, the findings of this jury may have been further enriched by expanding the scope of the topic to encompass the whole-of-hospital system. However, a larger scope would likely have been challenging to manage in a single jury process, particularly given juror feedback requesting longer deliberation time.

Citizens' Juries were found to be an effective method of engaging members of the public in decision-making on the complex issues such as the improvement of EDs. The study extends our understanding of public views on optimising access to and provision of emergency care and provides clear recommendations for direct public input to guide health policy. The jury's findings suggest that the public may be supportive of a 
multifaceted approach and may be open to flexible models of emergency health service delivery to combat ED crowding.

Acknowledgements The planning and implementation of this jury was informed by the work of The Jefferson Center for New Democratic Processes. Thanks are due to the witnesses and the members of the Citizens' Jury who gave their time to contribute to this research, Kylie Rixon and Anne Bucetti for their administrative support, and to the facilitators Max Hardy and Danielle Annells of Max Hardy consulting.

Contributors PAS, JAW, PB, EK, PL and AW conceived and designed the study, and obtained funding. PAS, PB, EK, RK and AW undertook the study; PAS, PB and EK presented the background and functioning of a citizen jury to the jurors, and AW and GF were independent content experts. RK undertook additional data collection from participants, supervised by EK and PAS. NM undertook data analysis and prepared a draft of this manuscript and all authors provided substantial input to this version of the manuscript.

Funding This research was supported by funding from an Australian Research Council Linkage Grant (\#LP100200446), and Partner Organisations Queensland Health (Metro South Hospitals and Health Service), Southern Adelaide Local Health Network, Inc. and the National Institute for Health and Care Excellence (UK). RK received a full scholarship from this ARC grant plus a top-up grant for 2014 from the Australian Centre for Health Services Innovation (PostGraduate Scholarship PHD-000432, funded round 1-2013). PL is supported by the National Institute for Health Research (NIHR) Collaboration for Leadership in Applied Health Research and Care South London at King's College Hospital NHS Foundation Trust. The researchers were independent from the funders and all authors had full access to all of the data (including statistical reports and tables) in the study and can take responsibility for the integrity of the data and the accuracy of the data analysis.

Competing interests PAS, NM, RK, PB, JAW, and EK report grants from Australian Research Council Linkage Grant, grants and non-financial support from Queensland Health, grants and non-financial support from Southern Adelaide Local Health Network, Inc., during the conduct of the study. RK reports personal fees from Australian Research Council Linkage Grant and the Australian Centre for Health Services Innovation, during the conduct of the study.

Ethics approval Griffith University and Flinders' University Human Research Ethics Committees (MED/09/12/HREC; 6088 SBREC).

Provenance and peer review Not commissioned; externally peer reviewed.

\section{REFERENCES}

1 Pines JM, Hilton JA, Weber EJ, et al. International perspectives on emergency department crowding. Acad Emerg Med 2011;18:1358-70.

2 Australasian College for Emergency Medicine. Statement on access block. S127. Melbourne, 2014. https://www.acem.org.au/getattachment/8c29d36e-27e2-40e79a1e-dfe73c5298ba/S127-Statement-on-Access-Block.aspx (accessed 14 June 2016).

3 Di Somma S, Paladino L, Vaughan L, et al. Overcrowding in emergency department: an international issue. Intern Emerg Med 2015;10:171-5.

4 Tregunno D, Rosss Baker G, Barnsley J, et al. Competing values of emergency department performance: Balancing multiple stakeholder perspectives. Health Serv Res 2004;39(Pt 1):771-91.
5 OECD. Citizens as partners: Information, consultation and public participation in policy-making. Paris: Organisation for Economic Cooperation and Development, 2001.

6 Mooney GA. Handbook on Citizens' Juries with Particular Reference to Health Care 2010. http://www.newdemocracy.com.au/library/research-papers/165-a-handbookon-citizens-juries-with-particular-reference-to-health-care (accessed 14 June 2016)

7 Whitty JA, Burton P, Kendall E, et al. Harnessing the potential to quantify public preferences for healthcare priorities through citizens' juries. Int J Health Policy Manag 2014;3:57-62.

8 Menon D, Stafinski T. Engaging the public in priority-setting for health technology assessment: findings from a citizens' jury. Health Expect 2008;11: 282-93.

9 Moretto N, Kendall E, Whitty J, et al. Yes, the government should tax soft drinks: findings from a citizens' jury in Australia. Int J Environ Res Public Health 2014;11:2456-71.

10 National Health and Hospitals Reform Commission. A Healthier future for all Australians: Final Report. Canberra: Australian Government, 2009.

11 Queensland Legislation, Office of the Queensland Parliamentary Counsel. Hospital and Health Boards Act. 2011. https://www.legislation.qld.gov.au/LEGISLTN/ CURRENT/H/HHNA11.pdf (accessed 14 Jun 2016).

12 The Jefferson Center. Citizens Jury Handbook. Secondary Citizens Jury Handbook, 2004. http://jefferson-center.org/ (accessed 16 Nov 2015).

13 Davies $C$, Wetherell M, Barnett E, et al. Opening the box: evaluating the Citizens Council of NICE. London: School of Health \& Social Welfare and Psychology Discipline, The Open University, 2005.

14 Krinks R, Kendall E, Whitty JA, et al. Do consumer voices in health-care citizens' juries matter? Health Expect 2015. (awaiting publication).

15 Scuffham PA, Ratcliffe J, Kendall E, et al. Engaging the public in healthcare decision-making: quantifying preferences for healthcare through citizens' juries. BMJ Open 2014;4;e005437.

16 Australian Bureau of Statistics. Labour Force, Australia, May 2012. Cat. No. 6202.0 Canberra: ABS, 2012.

17 Private Health Insurance Administration Council. Quarterly Statistics March 2012. Canberra: PHIAC, 2012.

18 Australian Bureau of Statistics. Education and work, Australia, May 2011 Cat. No. 62270D0001_201105. Canberra: ABS, 2011. https://www.humanservices.gov.au/ sites/default/files/documents/2011-03-concession-cards.pdf (accessed 14 June 2016).

19 Department of Education Employment and Workplace Relations. Employment by Occupation, May 2012. Secondary Employment by Occupation, May 2012. 2012. http://www.deewr.gov.au/LMIP/default.aspx?LLMIP/LFR/QLD_LFR_Occupation_Total (accessed 5 Jun 2015).

20 Australasian College for Emergency Medicine. Background paper: Access block. S127. Melbourne, 2014. https://www.acem.org.au/getattachment/febe70cf-185c4267-b6de-5dd1768b4ad3/S127-Background-Paper-Access-Block.aspx (accessed 14 Jun 2016).

21 Forero R, Hillman K. Access block and overcrowding: a literature review. Sydney: University of New South Wales, 2008.

22 FitzGerald G, Toloo GS, Aitken P, et al. Public use and perceptions of emergency departments: a population survey. Emerg Med Australas 2015;27:336-42.

23 Australian Institute of Health and Welfare. Australian hospital statistics 2013-2014: Emergency department care. Cat. no. HSE 153. Canberra, 2015.

24 Fitzgerald G, Toloo GS, Romeo M. Emergency healthcare of the future. Emerg Med Australas 2014;26:291-4. 